Reprod. Nutr. Dévelop., 1988, 28 (6 B), 1673-1680.

\title{
Notions récentes concernant le corps jaune
}

\author{
P. LEYMARIE, Annie BENHAÏM
}

Laboratoire de Biochimie, CNRS UA 609 GS 79

Faculté de Médecine, 14032 Caen, France.

\section{Summary. Recent acquisitions concerning the corpus luteum.}

Corpora lutea from sheep and cows as well as human and primates contain both large and small steroïdogenic cells exhibiting distinct functional properties. Only the small cells seem to be able to respond in vitro to LH stimulation by raising their progesterone secretion. However, the entire progesterone secretion of the corpus luteum has been shown to be regulated in vivo by $\mathrm{LH}$ in the primate.

The LH steroidogenic action involves the activation of membrane adenylate cyclase whose molecular mechanism has been elucidated. Then a rise in intracellular cyclic AMP induces phosphorylation by a cyclic AMP dependent protein kinase of steroïdogenic protein targets which have not yet been completely identified

In sheep and cows, luteolysis is believed to be the consequence of a series of reciprocal interactions between the corpus luteum whose large cells secrete pulses of oxytocin in response to $\mathrm{PGF}_{2 \alpha}$ luteolysin and the endometrium which secretes pulses of $\mathrm{PGF}_{2 \alpha}$ in response to oxytocin. The secretion of endometrial $\mathrm{PGF}_{2 \alpha}$ can only begin after the induction of endometrial receptors by estradiol, from the preovulatory follicles.

Similarly in women and primates luteolysis, which does not require the presence of the uterus, could be the consequence of local reciprocal paracrine interactions between luteal cells of different types. These interactions are likely to involve $\mathrm{PGF}_{2 x}$, oxytocin and estradiol.

The biochemical mechanism responsible for the inhibition by $P G F_{2 \alpha}$ of $\mathrm{LH}$ induced progesterone secretion in luteal cells could involve a stimulation in the cell membrane of protein kinase $\mathrm{C}$ and the rise of cytosolic $\mathrm{Ca}^{++}$.

Several peptides with high potential biological activity have been demonstrated to be present in, and/or secreted by, the corpus luteum in various species. It is too early to determine whether they are really involved in corpus luteum regulation.

Le rôle du corps jaune dans la sécrétion de la progestérone (associée chez les primates aux estrogènes), et par là-même dans l'installation et le maintien de la gestation, est connu depuis fort longtemps. On sait que la fonction lutéale dépend grandement du nombre et du degré de maturité des cellules folliculaires qui sont à l'origine du tissu lutéal et qui ont pratiquement cessé de se multiplier après leur lutéinisation. En revanche on connaît mal les mécanismes intimes de la régulation du corps jaune constitué et en particulier ceux qui gouvernent la régression spontanée du corps jaune ou lutéolyse.

On commence cependant à entrevoir la complexité de ces phénomènes dont l'étude a fait l'objet d'innombrables travaux analysés dans plusieurs revues récentes (Khan-Dawood et Dawood, 1986 ; Auletta et Flint, 1988 ; Niswender et Nett, 1988 ; Tsafriri, 1988). 


\section{Le corps jaune est constitué de plusieurs types cellulaires}

Le corps jaune n'est pas un organe homogène. II renferme au moins deux types de cellules stéroïdogènes qui se distinguent d'emblée par leur taille et comme nous le verrons plus loin par leurs fonctions. On savait depuis fort longtemps que chez la femme et chez les primates les petites cellules lutéales provenant de la thèque et les grandes provenant de la granulosa restent groupées dans des zones bien définies à l'intérieur du corps jaune. Dans les autres espèces où le corps jaune est plus homogène sur le plan histologique et notamment chez les ruminants et le porc on a montré également l'existence de grandes et de petites cellules qui se distinguent les unes des autres sur le plan fonctionnel (Lemon et Loir, 1977 ; Ursely et Leymarie, 1979 ; Fitz et al., 1982). L'étude de déterminants antigéniques de surface indiquent que les grandes cellules proviennent de la granulosa du follicule ovulatoire et les petites cellules de la thèque (Allila et Hansel, 1984). Au cours de la maturation du corps jaune certaines petites cellules se transforment apparemment en grandes cellules. Au cours de la gestation les déterminants antigéniques qui permettaient de les distinguer disparaissent.

\section{La gonadotrophine $L H$ contrôle la sécrétion de progestérone}

On a pu démontrer chez le primate la dépendance stricte du corps jaune vis-à-vis de la gonadotrophine $\mathrm{LH}$. Chez des animaux chez lesquels les sécrétions endogènes de LH avaient été abolies par lésion hypothalamique, l'injection pulsatile de LHRH maintient la sécrétion de progestérone qui cesse lorsqu'on interrompt l'administration de $\mathrm{LHRH}$ et se rétablit dès que l'on reprend l'injection (Hutchinson et Zeleznick, 1985).

En étudiant les taux circulants physiologiques de $\mathrm{LH}$ au cours du cycle menstruel on a observé que le rythme et l'amplitude des pics de LH diminuent en phase lutéale tardive (Ellinwood et al., 1984). On a pu démontrer cependant que cette diminution est insuffisante pour induire la lutéolyse dont il faut donc chercher ailleurs l'origine (Hutchinson et al., 1986). Des études effectuées chez la vache (Ursely et Leymarie, 1979) et la brebis (Fitz et al., 1982) ont montré que les petites cellules lutéales étaient presque totalement dépendantes in vitro de la présence de l'hormone LH pour leur sécrétion de progestérone alors que les grandes cellules étaient pratiquement insensibles à $\mathrm{LH}$ mais sécrétaient des quantités appréciables de progestérone en l'absence de stimulation.

De même, chez la femme, petites et grandes cellules ont pu être récemment séparées (Ohara et al., 1987). Les deux types cellulaires synthétisent in vitro de la progestérone, des estrogènes et des androgènes mais seules les petites cellules semblent sensibles à l'hormone LH.

Notons que l'existence de cellules lutéales sécrétant des quantités appréciables de progestérone, mais insensibles à l'hormone $\mathrm{LH}$, contraste singulièrement avec la dépendance stricte in vivo du corps jaune vis-à-vis de LH que nous avons mentionnée. L'existence d'interactions cellulaires permettant à $\mathrm{LH}$ de contrôler directement ou indirectement la totalité de la synthèse de progestérone pourrait expliquer ce paradoxe apparent. 
Le mécanisme moléculaire de l'action stéroïdogène de $L H$ n'est pas totalement élucidé.

On sait qu'après liaison à ses récepteurs, situés à la face externe des membranes plasmiques l'hormone LH provoque la stimulation de l'adenyl cyclase qui transforme en AMP cyclique I'ATP intra-cellulaire. La mécanique moléculaire de cette activation a été élucidée. Elle fait intervenir un facteur peptidique intra-membranaire, le complexe Ns qui appartient au groupe des «GTP protéines " car l'une de ses sous-unités polypeptidiques possède la propriété de lier et d'hydrolyser le GTP. Par liaison à son récepteur intracellulaire I'AMP cyclique active la protéine kinase $A$, enzyme cytoplasmique qui phosphoryle un certain nombre de substrats protéiques dans la cellule. Les cibles de cette enzyme responsables de la régulation de l'étape limitante mitochondriale de la stéroïdogenèse (le clivage de la chaîne latérale du cholestérol) n'ont pas encore été totalement identifiées mais l'hypothèse la plus couramment admise est celle d'une stimulation du transfert des molécules de cholestérol vers la membrane interne de la mitochondrie.

L'effet de LH se termine quand les récepteurs liés à la gonadotrophine sont internalisés par un phénomène d'endocytose qui fait intervenir le cytosquelette et aboutit à la séparation de l'hormone et de son récepteur dans les lysosomes avec recyclage d'un certain nombre de récepteurs restés intacts vers la membrane plasmique de la cellule (Niswender et al., 1985).

En cas de gestation, chez le primate et la femme, l'hormone gonadotrophine chorionique (CG) se lie au récepteur de LH et empêche la lutéolyse. Son action est beaucoup plus prolongée dans le temps que celle de LH car l'internalisation du complexe CG-récepteur n'intervient quaprès un temps environ 50 fois plus long que dans le cas de l'hormone LH.

\section{Rôle de la prolactine}

La prolactine qui exerce une action gonadotrope majeure sur le corps jaune de rat ne semble pas impliquée dans la régulation du corps jaune constitué chez les ruminants et les primates, et c'est semble-t-il indirectement, en affectant la maturation du follicule ovulatoire qu'une hyperprolactinémie est susceptible d'altérer la fonction lutéale chez la femme.

\section{$P G F_{2 \alpha}$ agent lutéolytique}

II est maintenant bien démontré que, chez les ruminants, la lutéolyse est induite par une lutéolysine d'origine utérine qui a été identifiée à la prostaglandine $F_{2 \alpha}$ laquelle est apparemment transférée chez la brebis de la veine utérine à l'artère ovarienne qui lui est accolée par un mécanisme de transfert à contre-courant. Chez les primates et chez la femme, à la différence de ce qui se passe chez les ruminants l'ablation de l'utérus n'empêche pas la lutéolyse. Cependant, dans ces espèces le corps jaune possède des récepteurs pour $\mathrm{PGF}_{2 \alpha}$ et synthétise cette substance que l'on retrouve dans la veine ovarienne en plus grande quantité durant la deuxième partie de la phase lutéale (Auletta et al., 1984). Administrée 
par voie générale, la $\mathrm{PGF}_{2 \alpha}$ est immédiatement lutéolytique chez les ruminants mais ne peut induire qu'une faible diminution de la progestéronémie chez les primates. Elle semble plus efficace si on l'injecte directement dans le corps jaune. La $P G F_{2 \alpha}$ d'origine ovarienne pourrait donc jouer chez les primates et la femme le rôle de la $\mathrm{PGF}_{2 \alpha}$ d'origine utérine chez les ruminants.

\section{Mécanisme d'action de $P G F_{2 \alpha}$}

Le mécanisme d'action de $P G F_{2 \alpha}$ n'est pas connu. Deux hypothèses non exclusives l'une de l'autre ont été proposées. L'une concerne une action directe de $\mathrm{PGF}_{2 \alpha}$ sur la cellule lutéale l'autre une réduction du débit sanguin dans le corps jaune. L'effet direct sur la cellule lutéale est particulièrement net in vitro dans le cas de l'ovaire de rat superovulé où l'on a pu montrer à la fois une diminution de la synthèse d'AMP cyclique induite par $\mathrm{LH}$ et une inhibition de l'action stéroïdogène de l'AMP cyclique (Thomas et al., 1978). Ces effets inhibiteurs ont également été retrouvés chez les ruminants (Fletcher et Niswender, 1982; Benhaïm et al., 1987a). Des travaux récents suggèrent que la prostaglandine $F_{2 \alpha}$ pourrait exercer ses effets directs sur la cellule lutéale par l'intermédiaire d'une élévation du taux de $\mathrm{Ca}^{++}$intra-cellulaire et l'activation éventuelle de la protéine kinase $\mathrm{C}$, enzyme membranaire «phospholipide et calcium dépendante » mise en jeu dans un certain nombre de transductions de signaux inter-cellulaire (Benhaïm et al., 1987b). Il a été récemment démontré que $P G F_{2 \alpha}$ stimule, dans la cellule lutéale bovine, la production d'inositol triphosphate $\left(\mathrm{IP}_{3}\right)$ connu pour induire la libération dans le cytosol du calcium stocké dans le réticulum endoplasmique (Davis et al., 1987a).

Chez les primates et chez la femme l'effet lutéolytique in vitro de PGF $\mathrm{P}_{2 \alpha}$ n'apparaît que pour des tissus prélevés au milieu et à la fin de la phase lutéale. Si I'on utilise du tissu prélevé dans la première moitié de la phase lutéale, l'effet inhibiteur de $\mathrm{PGF}_{2 \alpha}$ ne se manifeste qu'en présence de noradrénaline (Bennegard et al., 1984) ce qui souligne la possibilité d'une intervention simultanée de plusieurs régulateurs dans le phénomène de lutéolyse. De même le fait que l'action in vitro de la prostaglandine $\mathrm{F}_{2 \alpha}$ soit plus difficile à mettre en évidence sur des cellules isolées que sur des tranches de tissus suggère l'intervention de facteurs paracrines et d'interactions cellulaires.

Il convient de rappeler ici le rôle probable de l'estradiol intra-lutéal dont l'intervention dans le déclenchement de la lutéolyse chez les primates a été mis en évidence (Butler et al., 1975 ; Auletta et al., 1985).

II existe par ailleurs un facteur d'inhibition de la liaison de LH à ses récepteurs (LHRBI) détecté dans le corps jaune de plusieurs espèces et dont le taux augmente au cours de la phase lutéale chez la femme (Nakano et al., 1985).

Un autre agent lutéolytique potentiellement important chez les primates est l'ocytocine, produit de sécrétion du corps jaune des ruminants, présent également dans le corps jaune des primates et de la femme.

\section{Intervention de l'ocytocine dans la lutéolyse}

Chez les ruminants et notamment chez la brebis, l'ocytocine est synthétisée et sécrétée en abondance par les grandes cellules lutéales du corps jaune 
cyclique. Elle disparaît dans le corps jaune de gestation. En fin de phase lutéale l'estradiol sécrété par les follicules ovulatoires en croissance induit l'apparition de récepteurs à l'ocytocine dans l'endomètre utérin (Sheldrick et Flint, 1985).

L'ocytocine déclenche alors dans ce tissu un pulse de sécrétion de prostaglandine $F_{2 \alpha}$ qui exerce une double action sur le corps jaune : d'une part inhibition de la sécrétion de progestérone et d'autre part déclenchement d'un pulse de sécrétion d'ocytocine d'où une série d'interactions entre utérus et corps jaune qui aboutissent à la lutéolyse (Flint et Sheldrick, 1982).

Chez les primates et la femme, chez lesquels l'utérus n'intervient pas dans la lutéolyse, on a mis en évidence la synthèse d'une faible quantité d'ocytocine dans certaines cellules lutéales (Khan-Dawood et al., 1984). Par analogie avec ce que l'on observe chez les ruminants, on a évoqué une intervention de l'ocytocine dans la lutéolyse chez ces espèces dans le cadre d'interactions réciproques de type paracrine entre les cellules lutéales qui secrètent de l'ocytocine et des cellules lutéales qui secréteraient de la $\mathrm{PGF}_{2 \alpha}$ (Auletta et Flint, 1988) mais l'existence réelle d'un tel système de régulation est encore loin d'être établie.

Certes on a pu observer in vitro un léger effet inhibiteur par de fortes doses d'ocytocine sur la synthèse de progestérone induite par LH dans les cellules lutéales humaines (Tan et al., 1982 ; Bennegard et al., 1987) mais ces résultats sont quelque peu controversés (Richardson et Masson, 1985) et la présence de récepteurs à l'ocytocine dans des cellules lutéales n'a pas encore été démontrée.

\section{Autres facteurs synthétisés par le corps jaune}

On sait que le corps jaune sécrète, outre les facteurs stéroïdiens ou non stéroïdiens déjà mentionnés toute une série de peptides à haute activité biologique potentielle.

La relaxine est la plus anciennement connue. Sa structure a été élucidée. Elle est sécrétée par le corps jaune en début de gestation et pourrait participer à la relaxation du myomètre utérin au moment de l'implantation (Schmidt et al., 1986 ; Weiss, 1984).

L'inhibine est un peptide du fluide foliculaire dont on a récemment déterminé la structure. Elle a été mise en évidence dans le corps jaune qui la synthétise puisqu'il contient son ARN messager (Davis et al., 1987b). Son taux plasmatique chez la femme atteint son maximum au cours de la phase lutéale (McKachlan et al., 1987).

Un facteur ovarien de type $L H R H$ décrit initialement chez le rat semble exister également dans le corps jaune humain et pourrait intervenir dans la régulation négative de la stéroïdogenèse et la lutéolyse (Aten et al., 1987). Des récepteurs à LHRH ont été mis en évidence dans le corps jaune humain (Popkin et al., 1983).

La prorénine, précurseur inactif de la rénine dont la source principale est rénale, est également synthétisé dans l'ovaire humain (Sealey, 1987). Sa synthèse par le corps jaune a été récemment établie (Lightman et al., 1988). Son taux plasmatique présente un maximum durant la période périovulatoire. On a évoqué son rôle possible dans une production locale d'angiotensine II dont on connaît le rôle angiogénique potentiel (Sealey, 1987). 
L'epidermal growth factor ( $E G F$ ). Sa présence a été démontrée par immunocytochimie dans le corps jaune humain (Khan-Dawood, 1987) où il possède des récepteurs spécifiques (Ayyagari et Khan-Dawood, 1987). Ajouté à des cultures de granulosa humaine lutéinisée il stimule la multiplication cellulaire et la synthèse de progestérone (Tapanainen et al., 1987).

Le facteur FRP (follicular regulatory protein) isolé par Di Zerega du liquide folliculaire. II a été mis en évidence par immunocytochimie dans le corps jaune humain. Son taux d'excrétion urinaire évolue parallèlement à la progestéronémie au cours du cycle menstruel ce qui suggère que le corps jaune en est la source principale (Katt et al., 1988).

Les dérivés de la proopiomélanocortine. Enkephaline et arginine vasopressine et leur ARN messager respectif ont été également mis en évidence dans le corps jaune (Ménézo et al., 1987 ; Clements et al., 1988).

En conclusion le rôle crucial de l'hormone LH pour la sécrétion de progestérone par le corps jaune est maintenant pleinement confirmé et les mécanismes de son action en partie élucidés.

Pour ce qui est de la lutéolyse nous disposons d'un modèle bien établi chez les ruminants dans lequel $\mathrm{PGF}_{2 \alpha}$, ocytocine et estradiol interviennent dans le cadre d'interaction réciproque entre utérus et ovaire. Dans le cas de la femme et des primates où l'utérus n'intervient pas dans la lutéolyse, il semble légitime d'envisager un mécanisme impliquant également $\mathrm{PGF}_{2 x^{\prime}}$ ocytocine et estradiol dans le cadre d'interactions paracrines intra-ovariennes entre cellules lutéales de différents types.

L'élucidation complète des mécanismes de régulation du corps jaune passe à l'évidence par un inventaire préalable du lieu de synthèse et de l'action des régulateurs paracrines potentiels qui ont pu être inventoriés.

$27^{e}$ Réunion de la Société francaise pour l'Etude de la Fertilité Paris, 29, 30 sept., $1^{\text {er }}$ oct. 1988.

\section{Références}

ALILA H. W., HANSEL W., 1984. Origin of different cell types in the bovine corpus luteum as characterized by specific monoclonal antibodies. Biol. Reprod., 31, 1015-1025.

ATEN R. F., POLAN M. L., BAYLESS R., BEHRMAN H., 1987. A Gonadotropin-releasing hormone $(\mathrm{GnRH})$-like protein in human ovaries : similarity to the $\mathrm{GnRH}$-like ovarian protein of the rat. J. clin. Endocrinol. Metab., 64, 1288-1293.

AULETTA F. J., FLINT A. P. F., 1988. Mechanisms controlling corpus luteum function in sheep, cows, non human primates and women, especially in relation to the time of luteolysis. Endocr. Rev., 9, 88-106.

AULETTA F. J., KAMPS D. L., WESLEY M., GIBSON M., 1984. On Luteolysis in the Rhesus monkey ovarian venous estrogen, progesterone and prostaglandins $\mathrm{F}_{2 \alpha}$ metabolites. Prostaglandins, 27, 299-310.

AYYAGARI R. R., KHAN-DAWOOD F. S., 1987. Human corpus luteum : presence of epidermal growth factor receptors and binding characteristics. Am. J. Obst. Gynecol., 156, 942-946.

BENHAIIM A., HERROU M., MITTRE H., LEYMARIE P., 1987a. Effects of phorbol esters on steroidogenesis in small bovine luteal cells. FEBS Letters, 223, 321-326. 
BENHAIMM A., BONNAMY P. J., PAPADOPOULOS V., MITTRE H., LEYMARIE P., 1987b. In vitro action of $\mathrm{PGF}_{2 x}$ on progesterone and cAMP synthesis in small bovine luteal cells. Prostaglandins, 33, 227-239

BENNEGARD B., DENNEFORS B., HAMBERGER L., 1984. Interaction between catecholamines and prostaglandins $\mathrm{F}_{2 \alpha}$ in Human luteolysis. Acta endocrinol. (Copenh), 106, 532-537.

BENNEGARD B., HAHLIN M., DENNEFORS B., 1987. Antigonadotropic effect of oxytocin on the isolated human corpus luteum. Fertil. Steril., 47, 431-435.

BUTLER W. R., HOTCHKISS J., KNOBIL E., 1975. Functional luteolysis monkey : ovarian estrogen and progesterone during the luteal phase of the menstrual cycle. Endocrinology, 96, $1509-1513$

CLEMENTS J. A., LI H., RISBRIFDGER G., CHENG M. C., SMITH A. I., FUNDER J. W., 1988. Pituitary peptides in the ovary and testis. 8th int. Congr. Endocrinol., Kyoto, Abstr. S64.

DAVIS J. S., WEAKLAND L. L., WEILAND D. A., FARESE R. V., WESTL A., 1987a. Prostaglandin $\mathrm{F}_{2 \alpha}$ stimulates phosphatidyl-inositol 4,5-biphosphate hydrolysis and mobilizes intracellular calcium in bovine luteal cells. Proc. nat. Acad. Sci. USA, 84, 3728-3732.

DAVIS S. R., KROZOWSKI Z., MCLACHLAN R. I., BURGER A. G., 1987b. Inhibin gene expression in the human corpus luteum. J. Endocrinol., 115, R21-R23.

ELLINWOOD W. E., NORMAN R. L., SPIES H. G., 1984. Changing frequency of pulsatile LH and progesterone secretion during the luteal phase of the menstrual cycle of Rhesus monkey. Biol. Reprod., 31, 714-722.

FITZ T. A., MAYAN M. H., SAWYER H. R., NISWENDER G. D., 1982. Characterization of two steroidogenic cell types in the ovine corpus luteum. Biol. Reprod., 27, 703-711.

FLETCHER P. W., NISWENDER G. D., 1982. Effect of PGF $_{2 x}$ on progesterone secretion and adenylate cyclase activity in ovine luteal tissue. Prostaglandins, 23, 803-818.

FLINT A. P. F., SHELDRICK E. L., 1982. Ovarian secretion of oxytocin is stimulated by prostaglandins. Nature, 297, 587-588.

HUCHINSON J. S., ZELEZNICK A. J., 1985. The corpus luteum of the primate menstrual cycle is capable of recovering from a transient withdrawal of pituitary gonadotropin support. Endocrinology, 117, 1043-1050.

HUCHINSON J. S., NELSON P. B., ZELEZNICK A. J., 1986. Effect of different gonadotropin pulse frequences on corpus luteum function during the menstrual cycle of rhesus monkeys. Endocrinology, 119, 1964-1972.

KATT E., FUJIMORI K., YANAGIHARA D., CAMPEAU J., NUMAZAKI, HOLST P., TONETTA S., RODGERS K., WESTHOF G., MISHELL D., HORENSTEIN J., di ZEREGA G., 1988 . Determination of follicle regulatory protein levels in urine during the normal menstrual cycle using an enzyme-linked immunosorbant assay. J. clin. Endocrinol. Metab., 66, 1213-1219.

KHAN-DAWOOD F. S., MARUT E. L., DAWOOD M. Y., 1984. Oxytocin in the corpus luteum of the cytomologus monkey (Macaca facicularis). Endocrinology, 115, 570-574.

KHAN-DAWOOD F. S., 1987. Human corpus luteum : immunocytochemical localization of epidermal growth factor. Fertil. Steril., 47, 916-919.

KHAN-DAWOOD F. S., DAWOOD M. Y., 1986. Paracrine regulation of luteal function. Clinics Endocrinol. Metab., 15, 172-184.

LEMON M., LOIR M., 1977. Steroid release in vitro by two luteal cell types in the corpus luteum of the pregnant cow. J. Endocrinol., 75, 351-359.

LIGHTMAN A., RZAZA P. J., CULLER M. D., JONES C., CARIDE V. J., NEGRUVILAR A. F., DESCHEPPER C., DECHERNEY A. H., NAFTOLIN F., 1988. The ovarian renin angiotensin system (OVRAS). 8th int. Congr. Endocrinol., Kyoto, Abstr. $\$ 170$.

McLACHLAN R. I., RUBERTSON D. M., HEALY D. L., BURGER H. G., de KRETSER D. M., 1987. Circulating immunoreactive inhibin levels during the normal human menstrual cycle. J. clin. Endocrinol. Metab., 65, 954-962.

MENEZO Y., CUPO A., BUĖNO L., 1987. Production d'enképhalines par le corps jaune humain et bovin. C.R. Acad. Sci. Paris, Sér. III, 304, 337-339.

NAKANO R., IWASAKI M., YAMOTO M., 1985. Luteinizing hormone receptor binding in aqueous extract of human corpus luteum. Gyneco/. Obstet. Invest., 20, 45-51. 
NISWENDER G. D., NETT, 1988. The corpus luteum and its control. 13, 489-526. In KNOBIL E., NEILL J., The physiology of reproduction. Raven Press New York.

NISWENDER G. D., SCHWALL R. H., FITZ T. A., FARIN C. E., SAWYER H. R., 1985. Regulation of luteal function in domestic ruminants : new concepts. Rec. Prog. Horm. Res., 41, $101-151$.

OHARA A., MORI T., TAU S., BAN C., NARIMOTO K., 1987. Functional differenciation in steroidogenesis of two types of luteal cells isolated from human corpora lutea of menstrual cycle. J. clin. Endocrinol. Metab., 66, 1192-1200.

POPKIN R., BRAMLEY T. A., CURRIE A., SHAW R. W., BAIRD D. T., FRASER H. M., 1983. Specific binding of luteinizing hormone releasing hormone to human luteal tissue. Biochem. Biophys. Res. Commun., 114, 750-756.

RICHARDSON M. C., MASSON G. M., 1985. Lack of direct inhibitory action of oxytocin in progesterone production by dispersed cells from human corpus luteum. J. Endocrinol., 104, 149-151.

SEALEY J. E., GLORIOSO N., ITSKUVITZ J., LARAGH J. H., 1987. Prorenin as a reproductive hormone. Am. J. Med., 81, $1041-1046$.

SCHMIDT C. L., BLACK V. H., SAROSI P., WEISS G., 1986. Progesterone and relaxin secretion in relation to the ultrastructure of human luteal cells in culture: effects of human chorionic gonadotropin. Am. J. Obst. Gynecol., 155, 1209-1219.

SHELDRICK E. L., FLINT A. P. F., 1985. Endocrine control of uterine oxytocin receptors in the ewe. J. Endocrinol., 106, 249-258.

TAN G. J. S., TWEEDALE R., BIGGS J. S. G., 1982. Oxytocin may play a role in control of human corpus luteum. J. Endocrinol., 95, 65-70.

TAPANAINEN J., LEINUNEN P. J., TAPANAINEN P., YAMAMOTO V., JAFF R. B., 1987. Regulation of human granulosa luteal cell progesterone production and proliferation by gonadotropins and growth factors. Fertil. Steril., 48, 576-580.

THOMAS J. P., DORFLINGER L. J., BEHRMAN H. R., 1978. Mechanism of the rapid antigonadotropin action of prostaglandins in the cultured luteal cells. Proc. Nat. Acad. Sci. USA, 75, $1344-1348$.

TSAFRIRI A., 1988. Local nonsteroidal regulators of ovarian function. 14, 527-565. In KNOBIL E., NEILL J., The physiology of reproduction. Raven Press, New York.

URSELY J., LEYMARIE P., 1979. Varying response to luteinizing hormone of two luteal cell types isolated from bovine corpus luteum. $J$. Endocrinol., 83, 303-310.

WEISS G., 1984. Significance of relaxin in the human, 285-295. In SAIRAM M. R., ATKINSON L. E., Gonadal proteins and peptides and their biological significance. Raven Press, New York. 